Research Article

\title{
Proliferative Glomerulonephritis: Risk Factor for Hypertension in Lupus
}

\author{
Mansour Mbengue $\mathbb{D}^{1},{ }^{1}$ Motula Latou Lot, ${ }^{2}$ Seynabou Diagne, ${ }^{3}$ and Abdou Niang ${ }^{1}$ \\ ${ }^{1}$ Department of Nephrology, Dalal Jamm University Hospital, Dakar, Senegal \\ ${ }^{2}$ Department of Nephrology, Aristide Le Dantec University Hospital, Dakar, Senegal \\ ${ }^{3}$ Department of Nephrology, Pikine Hospital, Dakar, Senegal
}

Correspondence should be addressed to Mansour Mbengue; mansourmbengue92@gmail.com

Received 31 December 2020; Accepted 1 April 2021; Published 15 April 2021

Academic Editor: Thereza Maria Magalhães Moreira

Copyright (c) 2021 Mansour Mbengue et al. This is an open access article distributed under the Creative Commons Attribution License, which permits unrestricted use, distribution, and reproduction in any medium, provided the original work is properly cited.

\begin{abstract}
Studies report a high prevalence of hypertension in lupus, reaching up to $74 \%$. The incidence of hypertension in SLE patients is increased with the severity of the kidney damage. This work was carried out with the objective of determining the prevalence of hypertension in lupus nephritis and to seek the existence of an association between the presence of a proliferative glomerulonephritis and hypertension. Patients and Methods. This was a case-control study, carried out in the nephrology department of the Aristide Le Dantec University Hospital in Dakar. All records of patients with lupus nephritis over a 10-year period, from January 01, 2007, to December 31, 2016, were included. Results. During the study period, out of 64 lupus nephritis records collected, 28 patients had hypertension, for a hospital prevalence of $43.75 \%$. The mean age of the patients was 30.64 years \pm 10.44 . There were 24 women and 4 men. The mean systolic blood pressure was $156 \mathrm{mmHg}(110-220)$ and the mean diastolic blood pressure was $100 \mathrm{mmHg}(80-130)$. The mean serum creatinine was $29.48 \mathrm{mg} / \mathrm{l} \pm 24.99$. The mean proteinuria was $4.50 \mathrm{~g} /$ $24 \mathrm{~h} \pm 2.87$. Hypertriglyceridemia was observed in one patient. Hypercholesterolemia was present in 3 patients. HDL levels were normal in all patients and elevated LDL levels were noted in all 4 patients. None of our patients had diabetes. Class III was found in 11 cases, class IV in 14 cases, pure class V in 2 cases, and class II in 1 case. Hypertension was associated with the presence of proliferative glomerulonephritis (odds ratio, $7.45 ; 95 \%$ CI, 1.9 to $29.1 ; p=0.002$ ). Conclusion. Hypertension is common in lupus nephritis. The presence of a proliferative glomerulonephritis is a risk factor for the development of arterial hypertension. Screening and adequate management of hypertension are essential for the prevention of the progression of chronic kidney disease in lupus.
\end{abstract}

\section{Introduction}

Several studies report a high frequency of hypertension in lupus, reaching up to $74 \%$ in some cohorts [1-4]. The incidence of hypertension in SLE patients is also increased with the severity of the kidney damage. Besides nephropathy, other factors are involved in the development of hypertension in lupus. These include activation of the renin angiotensin aldosterone system (RAAS) and immune dysregulation seen in patients with SLE. The presence of hypertension has several implications for the prognosis of lupus nephritis. Studies in the recent years have shown that the primary cause of death for SLE patients is no longer the autoimmune pathology itself, neither the infectious consequences of immunosuppressive treatments, but primarily cardiovascular pathologies [5].

Lupus is the cause of major and accelerated atherosclerosis, possibly through nephropathy but also through chronic inflammation and metabolic complications, immunosuppressive treatments, and corticosteroids. A recent metaanalysis clearly confirms that SLE patients have a cardiovascular risk almost twice that of the general population, this being much more marked for the youngest patients [6].

Hypertension is one of the important determinants of the progression of atherosclerosis in lupus. In addition, hypertension is recognized as one of the factors contributing 
to the progression of chronic kidney disease (CKD). Hypertension in lupus nephritis is not well described in subSaharan Africa. This work is carried out with the objective of determining the prevalence of hypertension in lupus nephritis and to seek the existence of an association between the presence of a proliferative glomerulonephritis and hypertension.

\section{Patients and Methods}

This was a case-control study carried out in the nephrology department of the Aristide Le Dantec University Hospital in Dakar. All records of patients with lupus nephritis over a 10year period, from January 01, 2007, to December 31, 2016, were included. The cases were chosen by taking all the files of patients who presented hypertension. The patients who did not have hypertension were the controls. Patients who had a proliferative glomerulonephritis were the exposed. The patients who did not present a proliferative glomerulonephritis were the unexposed. The diagnosis of lupus nephritis was made after a concordant renal puncture biopsy associated with proteinuria greater than $0.5 \mathrm{~g} / 24 \mathrm{~h}$ or with active urine sediment [7]. Proliferative glomerulonephritis was defined by classes III or IV associated or not with a class $\mathrm{V}$ according to the classification of the ISN/RPS of 2003 [7]. Hypertension was defined according to the definition of the World Health Organization [8]. For each patient retained, epidemiological, clinical, biological, histological, therapeutic, and evolutionary data were studied. Glomerular filtration rate (GFR) was estimated according to the MDRD formula (The Modification of Diet in Renal Disease). The evolving modalities were recorded after a 6-month followup. Complete or partial remission, relapse, and resistance were defined according to the 2012 European League against Rheumatism and European Renal Association, European Dialysis and Transplant Association (EULAR/ERA/EDTA) criteria [9]. The data were collected on a preestablished form. They were entered with Sphinx software version 5.1.0.2. The data analysis was carried out with the SPSS software (Statistical package for Social Sciences) version 18. The descriptive study was carried out with the calculation of the frequencies and proportions for the qualitative variables and the calculation of the means, standard deviations, for the quantitative variables. The analytical study was done with cross tables. To compare the frequencies, we used Pearson's chi-square test or Fisher's two-tailed exact test; according to their conditions of applicability, the comparison of means was made with the analysis of variance test with a threshold of significance $p<0.05$. The $95 \%$ confidence interval of the odds ratio was calculated using the semi-exact method.

\section{Results}

During the study period, out of 64 lupus nephritis records collected, 28 patients were included, for a hospital prevalence of $43.75 \%$. The mean age of the patients was 30.64 years \pm 10.44 . There were 24 women and 4 men at a sex ratio of 0.16 . The mean systolic blood pressure was $156 \mathrm{mmHg}$ (110-220) and the mean diastolic blood pressure was
$100 \mathrm{mmHg}(80-130)$. The mean serum creatinine was $29.48 \mathrm{mg} / \mathrm{l} \pm 24.99$. The mean proteinuria was $4.50 \mathrm{~g} /$ $24 \mathrm{~h} \pm 2.87$. Hypertriglyceridemia was observed in one patient. Total hypercholesterolemia was present in 3 patients. HDL levels were normal in all patients and elevated LDL levels were noted in all 4 patients (Table 1). None of our patients had diabetes. Class III was found in 11 cases, class IV in 14 cases, pure class V in 2 cases, and class II in 1 case. In the induction treatment of patients with proliferative glomerulonephritis, cyclophosphamide was used in 12 cases, mycophenolate mofetil in 4 cases, and azathioprine in 1 case. This immunosuppressive treatment was associated with corticosteroid therapy in all cases. In the patient with class $\mathrm{V}+\mathrm{II}$, cyclophosphamide, combined with corticosteroid therapy, was used. After 6-month follow-up, there were 5 lost to follow-up. Of the remaining 23 patients, 6 were in complete remission, 6 were in partial remission, and 11 were resistant. There are 5 patients who had developed end-stage renal disease (ESRD). Death was observed in 4 patients and the causes were pulmonary embolism in one patient, bacterial meningitis in one patient, and pulmonary tuberculosis in one patient. Hypertension was associated with the presence of proliferative glomerulonephritis (odds ratio, 7.45; 95\% CI, 1.9 to 29.1; $p=0.002$ ) (Table 2).

\section{Discussion}

The association of lupus nephritis and hypertension is well reported $[10,11]$. Several studies have reported the high incidence of hypertension in lupus as high as 74\% [1-4]. In our series, $43.75 \%$ of patients had hypertension. In subSaharan Africa, studies found a prevalence ranging from $30 \%$ to $29.3 \%[12,13]$. On the other hand, the prevalence of hypertension was higher in the series of Contreras et al. [14] with $70 \%$ of cases, of Aoudia et al. [8] with $48.85 \%$ of cases, and of Martin-Gomez et al. [15] with 54.1\% of cases. Based on these results, it was found that the prevalence of hypertension in our study was lower than in studies done in white patients. This fact deserves a questioning because hypertension in general and particularly in patients with glomerulopathy is more frequent in black people because of the genetic predisposition linked to the presence of a polymorphism of the Apol 1 gene which codes for the apolipoprotein 1. More studies are needed to answer this question. This hypertension is multifactorial; glomerular involvement of lupus is the basis; it is worsened in the event of associated renal failure, especially since the patient is on corticosteroid therapy [15].

The incidence of hypertension in patients with lupus nephritis was also increased with both the severity of the kidney damage and the loss of kidney function. In our study, there is an association between hypertension and the presence of a proliferative glomerulonephritis. Okpechi et al. had also found this association between hypertension and the existence of a proliferative glomerulonephritis [13]. These results could be explained by the fact that cell proliferation leads to a decrease in GFR which will lead to renal failure and a decrease in urine output. The hypertension would be volo-dependent in relation to the hydrosodium 
TABLe 1: Comparison of socio-demographic, clinical characteristics of cases and controls.

\begin{tabular}{|c|c|c|c|}
\hline Variables & Hypertension $(n=28)$ & Without hypertension $(n=36)$ & $p$ value \\
\hline Mean age (years) & $30.64 \pm 10.45$ & $32.51 \pm 11.65$ & 0.51 \\
\hline Sex & & & 0.42 \\
\hline Women & $24(85.70 \%)$ & $28(77.70 \%)$ & \\
\hline Men & $4(14.30 \%)$ & $8(22.30 \%)$ & \\
\hline Serum creatinine $(\mathrm{mg} / \mathrm{L})$ & $29.48 \pm 24.99$ & $11.61 \pm 12.47$ & 0.01 \\
\hline Hemoglobin level (g/dL) & $8.80 \pm 2.48$ & $12.55 \pm 17.43$ & 0.27 \\
\hline Calcemia $(\mathrm{mg} / \mathrm{L})$ & $87.73 \pm 8.70$ & $91.35 \pm 7.80$ & 0.20 \\
\hline Phosphoremia (mg/L) & $56.50 \pm 38.78$ & $56.50 \pm 38.78$ & 0.32 \\
\hline Albuminemia $(\mathrm{g} / \mathrm{L})$ & $27.20 \pm 30.76$ & $22.21 \pm 7.74$ & 0.46 \\
\hline Protidemia $(g / L)$ & $57.23 \pm 9.47$ & $54.21 \pm 13.65$ & 0.42 \\
\hline Total cholesterol $(\mathrm{g} / \mathrm{L})$ & $2.95 \pm 0.61$ & $2.30 \pm 0.53$ & 0.13 \\
\hline HDL cholesterol (g/L) & $0.56 \pm 0.10$ & $0.52 \pm 0.20$ & 0.75 \\
\hline LDL cholesterol $(\mathrm{g} / \mathrm{L})$ & $2.04 \pm 0.48$ & $1.19 \pm 0.72$ & 0.09 \\
\hline Triglycerides $(\mathrm{g} / \mathrm{L})$ & $1.42 \pm 0.51$ & $1.35 \pm 0.84$ & 0.89 \\
\hline Proteinuria $(\mathrm{g} / 24 \mathrm{~h})$ & $4.50 \pm 2.87$ & $3.61 \pm 3.24$ & 0.26 \\
\hline Anti-DNA antibodies (\%) & $4(36.40)$ & $6(50.0)$ & 0.40 \\
\hline $\operatorname{GFR}\left(\mathrm{ml} / \mathrm{min} / 1.73 \mathrm{~m}^{2}\right)$ & $52.42 \pm 40.68$ & $121.48 \pm 59.60$ & \\
\hline
\end{tabular}

eGFR = estimated glomerular filtration rate according to 4-variable MDRD equation; HDL = high density lipoproteins; LDL = low density lipoproteins.

TABLE 2: Cross table of cases and controls.

\begin{tabular}{lccc}
\hline & Hypertension & Without hypertension & Total \\
\hline Proliferative glomerulonephritis & 25 & 19 & 44 \\
Without proliferative glomerulonephritis & 3 & 17 & 20 \\
Total & 28 & 36 & 64 \\
\hline
\end{tabular}

Odds ratio, $7.45 ; 95 \%$ CI, 1.9 to $29.1 ; p=0.002$.

retention linked to the decrease in urine output and to the reduction in oncotic pressure linked to hypoalbuminemia. Because of the kidney's importance in the long-term control of blood pressure, kidney damage may contribute to the development of hypertension in patients with SLE. Impaired renal hemodynamics and tubular function are the contributing factors since the kidney's ability to excrete sodium and water in response to changes in blood pressure is essential for effective long-term blood pressure control. Renal vascular dysfunction is a factor that may promote impaired renal hemodynamics. In addition, several studies have shown endothelial vascular dysfunction in lupus and impaired endothelial-dependent vasodilation and endothelial repair mechanisms in lupus patients [16].

However, hypertension associated with lupus can occur independently of the nephropathy [17]. For example, a recent study by Shaharir et al. found that $53 \%$ of patients with lupus in one cohort had hypertension despite the absence of renal impairment [18]. Other factors are involved in the onset of hypertension in lupus, including RAAS and immune dysregulation.

RAAS is also implicated because of its role in blood pressure balance and homeostasis. Angiotensin II can stimulate the production of endothelin-1 (ET-1), a peptide whose role in blood pressure control results from a balance between its vasoconstrictor and natriuretic actions in the renal medulla. Plasma ET-1 levels are increased in patients with lupus [19], and serum from lupus patients can induce ET-1 production by endothelial cells in vitro [20].
It is widely recognized that immune dysregulation and chronic inflammation have a role in the pathophysiology of hypertension in lupus. [21]. This is supported by the fact that treatment with the immunosuppressant mycophenolate mofetil in both animal models and in patients with essential hypertension reduced blood pressure [22]. Various inflammatory cytokines, including interleukins (IL-6, IL-17, and IL18), type I interferons, and tumor necrosis factor- $\alpha$ (TNF $\alpha$ ) have been implicated in the pathogenesis of lupus. Likewise, studies report a correlation between cytokines such as IL-6, $\mathrm{TNF} \alpha$, and C-reactive protein and blood pressure in patients with essential hypertension [23]. Inflammatory cytokines interact with important blood pressure regulators such as RAAS [24] and the sympathetic nervous system [25].

\section{Conclusion}

Hypertension is common in lupus nephritis. The presence of a proliferative glomerulonephritis is a risk factor for the onset of hypertension. Screening and adequate management of hypertension are essential for preventing the progression of chronic kidney disease in Lupus.

5.1. Limits. The limitation of our study was the lack of match on age and sex ratio between cases and controls. But we can see in our study that the cases and controls were comparable on age. Corticosteroid therapy could not be a confounding factor as hypertension was diagnosed before the patients were started on corticosteroids. 


\section{Data Availability}

The data used to support the findings of this study are included within the article.

\section{Ethical Approval}

All patient information was anonymised and written consent for publication of the study was obtained from the patients. The research was conducted ethically in accordance with the World Health Association Declaration of Helsinki and was approved by the local health ethics committee.

\section{Disclosure}

This research received no specific grant from any funding agency in the public, commercial, or not-for-profit sectors.

\section{Conflicts of Interest}

The authors have no conflicts of interest to declare.

\section{Authors' Contributions}

Mansour Mbengue reviewed the literature and wrote the first draft of the manuscript. All authors reviewed and edited the manuscript and approved the final version of the manuscript.

\section{References}

[1] A. Al-Herz, S. Ensworth, K. Shojania, and J. M. Esdaile, "Cardiovascular risk factor screening in systemic lupus erythematosus," Journal of Rheumatology, vol. 30, pp. 493496, 2003.

[2] B. F. Mandell, "Cardiovascular involvement in systemic lupus erythematosus," Seminars in Arthritis and Rheumatism, vol. 17, no. 2, pp. 126-141, 1987.

[3] M. Petri, "Detection of coronary artery disease and the role of traditional risk factors in the Hopkins Lupus Cohort," Lupus, vol. 9, no. 3, pp. 170-175, 2000.

[4] G. F. Mitchell, C.-Y. Guo, S. Kathiresan et al., "Vascular stiffness and genetic variation at the endothelial nitric oxide synthase locus," Hypertension, vol. 49, no. 6, pp. 1285-1290, 2007.

[5] M. G. Tektonidou, E. Kravvariti, G. Konstantonis, N. Tentolouris, P. P. Sfikakis, and A. Protogerou, "Subclinical atherosclerosis in systemic lupus erythematosus: comparable risk with diabetes mellitus and rheumatoid arthritis," Autoimmunity Reviews, vol. 16, no. 3, pp. 308-312, 2017.

[6] S. R. Schoenfeld, S. Kasturi, and K. H. Costenbader, "The epidemiology of atherosclerotic cardiovascular disease among patients with SLE: a systematic review," Seminars in Arthritis and Rheumatism, vol. 43, no. 1, pp. 77-95, 2013.

[7] Y. Asanuma, A. Oeser, A. K. Shintani et al., "Premature coronary-artery atherosclerosis in systemic lupus erythematosus," New England Journal of Medicine, vol. 349, no. 25, pp. 2407-2415, 2003.

[8] R. Aoudia, M. Omrane, H. Gaied et al., "Profil histologique et évolutif de l'hypertension artérielle chez les patients atteints d'une néphropathie lupique," Néph-Thér., vol. 13, pp. 296-310, 2017.
[9] I. N. Bruce, "Cardiovascular disease in lupus patients: should all patients be treated with statins and aspirin?" Best Practice and Research Clinical Rheumatology, vol. 19, no. 5, pp. 823838, 2005.

[10] D. R. Budman and A. D. Steinberg, "Hypertension and renal disease in systemic lupus erythematosus," Archives of Internal Medicine, vol. 136, no. 9, pp. 1003-1007, 1976.

[11] M. Nakano, M. Ueno, H. Hasegawa et al., "Renal haemodynamic characteristics in patients with lupus nephritis," Annals of the Rheumatic Diseases, vol. 57, no. 4, pp. 226-230, 1998.

[12] M. 1 Mansour, F. 1 Maria, L. T. 1 Ahmed et al., "Anatomoclinical aspects and progression of lupus nephritis in african patients," Nephrology and Urology, vol. 6, no. 1, p. 037, 2019.

[13] I. Okpechi, C. Swanepoel, N. Tiffin, M. Duffield, and B. Rayner, "Clinicopathological insights into lupus nephritis in South Africans: a study of 251 patients," Lupus, vol. 21, no. 9, pp. 1017-1024, 2012.

[14] G. Contreras, O. Lenz, V. Pardo et al., "Outcomes in african Americans and hispanics with lupus nephritis," Kidney International, vol. 69, no. 10, pp. 1846-1851, 2006.

[15] M. A. Martin-Gomez, M. A. Frutos Sanz, E. De Ramon Garrido et al., "Malaga study: 25 Year background in lupus nephritis in south of Spain," Lupus Open Access, vol. 1, p. 1, 2016.

[16] E. B. Taylor and M. J. Ryan, "Understanding mechanisms of hypertension in systemic lupus erythematosus," Therapeutic Advances in Cardiovascular Disease, vol. 11, no. 1, pp. 20-32, 2017.

[17] M. M. Ward and S. Studenski, "Clinical prognostic factors in lupus nephritis. the importance of hypertension and smoking," Archives of Internal Medicine, vol. 152, no. 10, pp. 2082-2088, 1992.

[18] S. S. Shaharir, R. Mustafar, R. Mohd, M. S. Mohd Said, and H. Gafor, "Persistent hypertension in lupus nephritis and the associated risk factors," Clinical Rheumatology, vol. 34, no. 1, pp. 93-97, 2015.

[19] H. Julkunen, O. Saijonmaa, C. Gronhagen-Riska, A. M. Teppo, and F. Fyhrquist, "Raised plasma concentrations of endothelin-1 in systemic lupus erythematosus," Annals of the Rheumatic Diseases, vol. 50, no. 7, pp. 526-527, 1991.

[20] T. Yoshio, J. Masuyama, A. Mimori, A. Takeda, S. Minota, and S. Kano, "Endothelin-1 release from cultured endothelial cells induced by sera from patients with systemic lupus erythematosus," Annals of the Rheumatic Diseases, vol. 54, no. 5, pp. 361-365, 1995.

[21] D. G. Harrison, A. Vinh, H. Lob, and M. S. Madhur, "Role of the adaptive immune system in hypertension," Current Opinion in Pharmacology, vol. 10, no. 2, pp. 203-207, 2010.

[22] J. Herrera, A. Ferrebuz, E. G. Macgregor, and B. RodriguezIturbe, "Mycophenolate mofetil treatment improves hypertension in patients with psoriasis and rheumatoid arthritis," Journal of the American Society of Nephrology, vol. 17, no. 12 suppl 3, pp. S218-S225, 2006.

[23] G. J. Blake, N. Rifai, J. E. Buring, and P. M. Ridker, "Blood pressure, C-reactive protein, and risk of future cardiovascular events," Circulation, vol. 108, no. 24, pp. 2993-2999, 2003.

[24] A. R. Brasier, A. Recinos, and M. S. Eledrisi, "Vascular inflammation and the renin-angiotensin system," Arteriosclerosis, Thrombosis, and Vascular Biology, vol. 22, no. 8, pp. 1257-1266, 2002.

[25] G. Pongratz and R. Straub, "The sympathetic nervous response in inflammation," Arthritis Research and Therapy, vol. 16, p. 504, 2014. 